\title{
Morita invariance of the filter dimension and of the inequality of Bernstein
}

\author{
V. V. Bavula and V. Hinchcliffe
}

\begin{abstract}
It is proved that the filter dimenion is Morita invariant. A direct consequence of this fact is the Morita invariance of the inequality of Bernstein: if an algebra $A$ is Morita equivalent to the ring $\mathcal{D}(X)$ of differential operators on a smooth irreducible affine algebraic variety $X$ of dimension $n \geq 1$ over a field of characteristic zero then the Gelfand-Kirillov dimension $\mathrm{GK}(M) \geq n=\frac{\mathrm{GK}(A)}{2}$ for all nonzero finitely generated $A$-modules $M$. In fact, a more strong result is proved, namely, a Morita invariance of the holonomic number for finitely generated algebra. As a direct consequence of this fact an affirmative answer is given to the question/conjecture posed by Ken Brown several years ago of whether an analogue of the inequality of Bernstein holds for the (simple) rational Cherednik algebras $H_{c}$ for integral c: $\mathrm{GK}(M) \geq n=\frac{\mathrm{GK}\left(H_{c}\right)}{2}$ for all nonzero finitely generated $H_{c}$-modules $M$.

Mathematics subject classification 2000: 16P90, 13N10, 16S32, 16P90, 16D30, $16 W^{r} 70$.
\end{abstract}

\section{Introduction}

Throughout the paper, $K$ is a field, a module $M$ over an algebra $A$ means a left module denoted ${ }_{A} M$, and GK $\left({ }_{A} M\right)$ is the Gelfand-Kirillov dimension of an $A$-module $M$.

Intuitively, the filter dimension of an algebra $\operatorname{fdim}(A)$ or a module fdim $(M)$ measures how 'close' standard filtrations of the algebra or the module are. In particular, for a simple algebra it also measures the growth of how 'fast' one can prove that the algebra is simple.

The filter dimension appears naturally when one wants to generalize the inequality of Bernstein for the ring $\mathcal{D}(X)$ of differential operators (as above) to the class of simple finitely generated algebras (recall that $\mathcal{D}(X)$ is a simple finitely generated algebra).

Theorem 1.1 (The inequality of Bernstein) Let $X$ be a smooth irreducible affine algebraic variety of dimension $n=\operatorname{dim}(X) \geq 1$ over a field of characteristic zero and $\mathcal{D}(X)$ be the ring of differential operators on $X$. Then

$$
\operatorname{GK}(M) \geq n=\frac{\operatorname{GK}(\mathcal{D}(X))}{2}
$$

for all nonzero finitely generated $\mathcal{D}(X)$-modules $M$. 
Theorem 1.2 [1] Let $A$ be a simple finitely generated algebra. Then

$$
\operatorname{GK}(M) \geq \frac{\operatorname{GK}(A)}{\operatorname{fdim}(A)+\max \{\operatorname{fdim}(A), 1\}}
$$

for all nonzero finitely generated $A$-modules $M$.

The following theorem is proved in Section 2

Theorem 1.3 (Morita invariance of the filter dimension) Morita equivalent simple finitely generated algebras have the same filter dimension.

As an immediate consequence one has the corollary.

Corollary 1.4 (Morita invariance of the inequality of Bernstein) Let $X$ and $\mathcal{D}(X)$ be as in Theorem 1.1. If the algebra $A$ is Morita equivalent to the ring of differential operators $\mathcal{D}(X)$ then

$$
\mathrm{GK}(M) \geq n=\frac{\mathrm{GK}(A)}{2}
$$

for all nonzero finitely generated $A$-modules $M$.

Proof. The algebra $\mathcal{D}(X)$ is a simple finitely generated algebra of Gelfand-Kirillov dimension $2 n$, hence the algebra $A$ is a simple finitely generated algebra of Gelfand-Kirillov dimension $2 n$ (since all the mentioned properties of algebra are Morita invariant and the algebra $A$ is Morita equivalent to $\mathcal{D}(X))$. The filter $\operatorname{dimension} f \operatorname{fdim}(\mathcal{D}(X))=1$, [2] By Theorem 1.3, $\operatorname{fdim}(A)=\mathrm{fdim}(\mathcal{D}(X))=1$, and by Theorem 1.2.

$$
\mathrm{GK}(M) \geq \frac{2 n}{1+1}=n
$$

for all nonzero finitely generated $A$-modules $M$.

Corollary 1.5 Let $\mathcal{D}\left(Q_{c}\right)$ be the ring of differential operators on quasi-invariants (over $\mathbb{C})$ for integral values of $c$ (see [4], [5], and [6]). Then $\mathrm{GK}(M) \geq n=\frac{\mathrm{GK}\left(\mathcal{D}\left(Q_{c}\right)\right)}{2}$ for all nonzero finitely generated $\mathcal{D}\left(Q_{c}\right)$-modules $M$.

Proof. The algebra $\mathcal{D}\left(Q_{c}\right)$ is Morita equivalent to the $n$ 'th Weyl algebra $A_{n}$, 4, and the result follows from Corollary 1.4 .

For an algebra $A$,

$$
h_{A}:=\inf \{\mathrm{GK}(M) \mid M \neq 0 \text { is a finitely generated } A-\text { module }\}
$$

is called the holonomic number of $A$. If $A$ is a simple finitely generated infinite dimensional algebra then $h_{A} \geq 1$. For the ring $\mathcal{D}(X)$ of differential operators on a smooth irreducible affine algebraic variety $X$ of dimension $n \geq 1$ over a field of characteristic zero, $h_{\mathcal{D}(X)}=n$. In particular, for the $n$ 'th Weyl algebra $A_{n}, h_{A_{n}}=n$. 
Theorem 1.6 (Morita invariance of holonomic number) Let $A$ and $B$ be Morita equivalent finitely generated algebras. Then $h_{A}=h_{B}$.

Proof. This follows from Theorem 1.7 since ${ }_{A} M \mapsto{ }_{B} Y \otimes_{A} M$ is the equivalence of the categories of $A$-modules and $B$-modules.

Theorem 1.7 Let $A$ and $B$ be Morita equivalent finitely generated algebras, and $\left(\begin{array}{cc}A & X \\ Y & B\end{array}\right)$ be a Morita context that provides the Morita equivalence $(X Y=A$ and $Y X=B$ where ${ }_{A} X_{B}$ and ${ }_{B} Y_{A}$ are bimodules (necessarily finitely generated on each side, [8], Sec. 3)). Then, for each finitely generated A-module $M, \operatorname{GK}\left({ }_{A} M\right)=\operatorname{GK}\left({ }_{B} Y \otimes_{A} M\right)$.

The next corollary gives an affirmative answer to the question/conjecture posed by Ken Brown several years ago (in his conference talk) of whether an analogue of the inequality of Bernstein holds for (simple) rational Cherednik algebras $H_{c}$ for integral $c$.

Corollary 1.8 Let $H_{c}$ be the rational Cherednik algebra over $\mathbb{C}$ associated to a finite Coxeter group $W$ in an $n$-dimensional vector space $h$ where $c$ is integral (see [4] for detail). Then $\operatorname{GK}(M) \geq n$ for all nonzero finitely generated $H_{c}$-modules.

Proof. The algebra $H_{c}$ is Morita equivalent to the cross product $A_{n} \# W$ where $A_{n}$ is the $n$ 'th Weyl algebra [4, hence $h_{H_{c}}=h_{A_{n} \# W}$ (Theorem 1.6). Since the action of the group $W$ on $A_{n}$ preserves the natural (standard) filtration of the Weyl algebra $A_{n}$ (by the total degree of the canonical generators $x_{i}$ and $\partial_{j}$ ), and the group $W$ is finite, it follows at once from definition of the Gelfand-Kirillov dimension that $h_{A_{n} \# W}=h_{A_{n}}=n$. Hence,

$$
\operatorname{GK}(M) \geq h_{H_{c}}=h_{A_{n} \# W}=h_{A_{n}}=n
$$

for all nonzero finitely generated $H_{c}$-modules $M$.

\section{The filter dimension and the holonomic number are Morita invariant}

The filter dimension. Let $\mathcal{F}$ be the set of all functions from the set of natural numbers $\mathbb{N}=\{0,1, \ldots\}$ to itself. For each function $f \in \mathcal{F}$, the non-negative real number or $\infty$ defined as

$$
\gamma(f):=\inf \left\{r \in \mathbb{R} \mid f(i) \leq i^{r} \text { for } i \gg 0\right\}
$$

is called the degree of $f$. The function $f$ has polynomial growth if $\gamma(f)<\infty$. Let $f, g, p \in \mathcal{F}$, and $p(i)=p^{*}(i)$ for $i \gg 0$ where $p^{*}(t) \in \mathbb{Q}[t]$ (a polynomial algebra with coefficients from the field of rational numbers). Then

$$
\begin{array}{rlr}
\gamma(f+g) \leq \max \{\gamma(f), \gamma(g)\}, & \gamma(f g) \leq \gamma(f)+\gamma(g), \\
\gamma(p)=\operatorname{deg}_{t}\left(p^{*}(t)\right), & \gamma(p g)=\gamma(p)+\gamma(g) .
\end{array}
$$


Let $A=K\left\langle a_{1}, \ldots, a_{s}\right\rangle$ be a finitely generated $K$-algebra. The finite dimensional filtration $F=\left\{A_{i}\right\}_{i \geq 0}$ associated with the algebra generators $a_{1}, \ldots, a_{s}$ :

$$
A_{0}:=K \subseteq A_{1}:=K+\sum_{i=1}^{s} K a_{i} \subseteq \cdots \subseteq A_{i}:=A_{1}^{i} \subseteq \cdots
$$

is called the standard filtration for the algebra $A$. Let $M=A M_{0}$ be a finitely generated $A$-module where $M_{0}$ is a finite dimensional generating subspace. The finite dimensional filtration $\left\{M_{i}:=A_{i} M_{0}\right\}$ is called the standard filtration for the $A$-module $M$.

Definition. $\operatorname{GK}(A):=\gamma\left(i \mapsto \operatorname{dim}_{K}\left(A_{i}\right)\right)$ and $\operatorname{GK}(M):=\gamma\left(i \mapsto \operatorname{dim}_{K}\left(M_{i}\right)\right)$ are called the Gelfand-Kirillov dimensions of the algebra $A$ and the $A$-module $M$ respectively. For more detail on the Gelfand-Kirillov dimension the reader is referred to the books [7], 8].

\section{The return functions and the (left) filter dimension.}

Definition [1]. The function $\nu_{F, M_{0}}: \mathbb{N} \rightarrow \mathbb{N} \cup\{\infty\}$,

$$
\nu_{F, M_{0}}(i):=\min \left\{j \in \mathbb{N} \cup\{\infty\}: A_{j} M_{i, g e n} \supseteq M_{0} \text { for all } M_{i, g e n}\right\}
$$

is called the return function of the $A$-module $M$ associated with the filtration $F=\left\{A_{i}\right\}$ of the algebra $A$ and the generating subspace $M_{0}$ of the $A$-module $M$ where $M_{i, g e n}$ runs through all generating subspaces for the $A$-module $M$ such that $M_{i, g e n} \subseteq M_{i}$.

Definition, [1. fdim $(M):=\gamma\left(\nu_{F, M_{0}}\right)$ is called the filter dimension of the $A$-module $M$, and $\operatorname{fdim}(A):=\operatorname{fdim}\left({ }_{A} A_{A}\right)$ is called the filter dimension of the algebra $A$ (note that ${ }_{A} A_{A} \simeq{ }_{A \otimes A^{o p}} A$ where $A^{o p}$ is the opposite algebra to $A$ ).

It is easy to check that the filter dimension does not depend neither on the choice of the filtration $F$ nor $M_{0}$.

Suppose, in addition, that the finitely generated algebra $A$ is a simple algebra. The return function $\nu_{F} \in \mathcal{F}$ and the left return function $\lambda_{F} \in \mathcal{F}$ for the algebra $A$ with respect to the standard filtration $F:=\left\{A_{i}\right\}$ for the algebra $A$ are defined by the rules:

$$
\begin{aligned}
& \nu_{F}(i):=\min \left\{j \in \mathbb{N} \cup\{\infty\} \mid 1 \in A_{j} a A_{j} \text { for all } 0 \neq a \in A_{i}\right\}, \\
& \lambda_{F}(i):=\min \left\{j \in \mathbb{N} \cup\{\infty\} \mid 1 \in A a A_{j} \text { for all } 0 \neq a \in A_{i}\right\},
\end{aligned}
$$

where $A_{j} a A_{j}$ is the vector subspace of the algebra $A$ spanned over the field $K$ by the elements $x a y$ for all $x, y \in A_{j}$; and $A a A_{j}$ is the left ideal of the algebra $A$ generated by the set $a A_{j}$. Under a mild restriction the maps $\nu_{F}(i)$ and $\lambda_{F}(i)$ are finite (see [3]). It is easy to check [1] that

$$
\operatorname{fdim}(A)=\gamma\left(\nu_{F}\right)
$$

More results on the filter dimension the reader can find in $[3]$.

Morita equivalence and Morita context. Let us recall some facts we need on Morita equivalence, for proofs the reader is refereed to [8]: if algebras $A$ and $B$ are Morita equivalent then $\mathrm{GK}(A)=\mathrm{GK}(B) ; A$ is a simple algebra iff $B$ is a simple algebra; $A$ is a finitely generated algebra iff $B$ is a finitely generated algebra. 
Algebras $A$ and $B$ are Morita equivalent iff there is a Morita context $\left(\begin{array}{ll}A & X \\ Y & B\end{array}\right)$ with $X Y=A$ and $Y X=B$ where ${ }_{A} X_{B}$ and ${ }_{B} Y_{A}$ are bimodules (necessarily finitely generated on each side, [8], Sec. 3).

\section{Proof of Theorem 1.3}

Let $A$ and $B$ be simple finitely generated Morita equivalent algebras. The algebras $A$ and $B$ are Morita equivalent, hence, by [8], 3.5.4, there is a Morita context $\left(\begin{array}{ll}A & X \\ Y & B\end{array}\right)$ with $X Y=A$ and $Y X=B$ where bimodules ${ }_{A} X_{B}$ and ${ }_{B} Y_{A}$ are finitely generated on each side, i.e. $X=A X_{0}=X_{0} B$ and $Y=B Y_{0}=Y_{0} A$ for some finite dimensional subspaces $X_{0} \subseteq X$ and $Y_{0} \subseteq Y$. Let $\left\{A_{i}\right\}$ and $\left\{B_{i}\right\}$ be standard filtrations on the algebras $A$ and $B$ respectively. Enlarging, if necessary, the spaces $X_{0}$ and $Y_{0}$ one may assume that there is a natural number $c$ such that

$$
A_{1} X_{0} \subseteq X_{0} B_{c}, \quad X_{0} B_{1} \subseteq A_{c} X_{0}, \quad B_{1} Y_{0} \subseteq Y_{0} A_{c}, \quad Y_{0} A_{1} \subseteq B_{c} Y_{0}
$$

The one-sided modules $X$ and $Y$ are equipped with following standard filtrations:

$$
\left\{X_{i}:=A_{i} X_{0}\right\},\left\{X_{i}^{\prime}:=X_{0} B_{i}\right\}, \quad\left\{Y_{i}^{\prime}:=B_{i} Y_{0}\right\}, \quad\left\{Y_{i}:=Y_{0} A_{i}\right\} .
$$

Recall that $\operatorname{fdim}(A)=\gamma(\nu)$ where

$$
\nu(i):=\min \left\{j \mid 1 \in A_{j} a_{i} A_{j} \text { for all } 0 \neq a_{i} \in A_{i}\right\},
$$

where $A_{j} a_{i} A_{j}$ means the vector subspace spanned by all the product $x a_{i} b$ for $x, y \in A_{j}$. Similarly, $\operatorname{fdim}(B)=\gamma\left(\nu^{\prime}\right)$ where $\nu^{\prime}$ is defined as above but for the algebra $B$ rather than $A$. For each $i \geq 0$,

$$
A_{i} X_{0} \subseteq X_{0} B_{c i}, \quad X_{0} B_{i} \subseteq A_{c i} X_{0}, \quad B_{i} Y_{0} \subseteq Y_{0} A_{c i}, \quad Y_{0} A_{i} \subseteq B_{c i} Y_{0}
$$

or, equivalently,

$$
X_{i} \subseteq X_{c i}^{\prime}, \quad X_{i}^{\prime} \subseteq X_{c i}, \quad Y_{i}^{\prime} \subseteq Y_{c i}, \quad Y_{i} \subseteq Y_{c i}^{\prime} .
$$

Here and everywhere the product $V_{1} \cdots V_{s}$ of subspaces in an algebra means the linear span of all the products $\left\{v_{1} \cdots v_{s} \mid v_{1} \in V_{1}, \ldots, v_{s} \in V_{s}\right\} . Y X=B$ and $X Y=A$ imply

$$
1_{B} \in B_{d} Y_{0} X_{0} B_{d} \text { and } 1_{A} \in A_{d} X_{0} Y_{0} A_{d}
$$

for some natural number $d$. We may also assume that $X_{0} Y_{0} \subseteq A_{d}$ and $Y_{0} X_{0} \subseteq B_{d}$. For each nonzero element $a \in A_{i}, 1_{A} \in A_{\nu(i)} a A_{\nu(i)}$, hence

$$
1_{B} \in B_{d} Y_{0} 1_{A} X_{0} B_{d} \subseteq B_{d} Y_{0} A_{\nu(i)} a A_{\nu(i)} X_{0} B_{d} \subseteq B_{c \nu(i)+d} Y_{0} a X_{0} B_{c \nu(i)+d} .
$$

For each $i \geq 0$ and each nonzero element $b \in B_{i}, X_{0} B_{d} b B_{d} Y_{0} \neq 0$ since, by (2),

$$
0 \neq b=1_{B} b 1_{B} \in B_{d} Y_{0} \cdot X_{0} B_{d} b B_{d} Y_{0} \cdot X_{0} B_{d} .
$$


Clearly,

$$
X_{0} B_{d} b B_{d} Y_{0} \subseteq X_{0} B_{i+2 d} Y_{0} \subseteq A_{c(i+2 d)} X_{0} Y_{0} \subseteq A_{c(i+2 d)+d} .
$$

Let $l(i):=c \nu(c i+2 c d+d)+d$. By (3) and (4), for each nonzero element $b \in B_{i}$,

$$
1_{B} \in B_{l(i)} Y_{0} \cdot X_{0} B_{d} b B_{d} Y_{0} \cdot X_{0} B_{l(i)} \subseteq B_{l(i)+2 d} b B_{l(i)+2 d} .
$$

Therefore,

$$
\nu^{\prime}(i) \leq c \nu(c i+2 c d+d)+3 d, \quad i \geq 0,
$$

hence $\operatorname{fdim}(B)=\gamma\left(\nu^{\prime}\right) \leq \gamma(\nu)=\operatorname{fdim}(A)$. By symmetry, we have the opposite inequality as well, and so $\mathrm{fdim}(A)=\mathrm{fdim}(B)$.

Proof of Theorem 1.7. We keep the notations of the proof of Theorem [1.3. Enlarging, if necessary, the spaces $X_{0}$ and $Y_{0}$ one may assume that $1_{A} \in X_{0} Y_{0}$ and $1_{B} \in Y_{0} X_{0}$. Let $M=A M_{0}$ be a finitely generated $A$-module $\left(\operatorname{dim}\left(M_{0}\right)<\infty\right)$ and $\left\{M_{i}:=A_{i} M_{0}\right\}$ be the standard filtration on $M$. The $B$-module $M^{\prime}:=Y \otimes_{A} M=B Y_{0} M_{0}$ is a finitely generated $B$-module with the finite dimensional generating space $Y_{0} M_{0}$ which is the image of $Y_{0} \otimes_{K} M_{0}$ under the map $\phi: Y \otimes_{K} M \rightarrow Y \otimes_{A} M$. Similarly, for vector subspaces $V \subseteq Y$ and $U \subseteq M$, $V U$ stands for $\phi\left(V \otimes_{K} U\right)$. Consider the standard filtration $\left\{M_{i}^{\prime}:=B_{i} Y_{0} M_{0}\right\}$ on the $B$ module $M^{\prime}$. Fix a natural number, say $e$, such that $\operatorname{dim}\left(X_{0}\right), \operatorname{dim}\left(Y_{0}\right) \leq e$. First, we prove that

$$
\frac{\operatorname{dim}\left(M_{i}\right)}{e} \leq \operatorname{dim}\left(Y_{0} M_{i}\right) \leq \operatorname{dim}\left(M_{c i}^{\prime}\right), \quad i \geq 0 .
$$

$1_{A} \in X_{0} Y_{0}$ implies $M_{i}=1_{A} M_{i} \subseteq X_{0} Y_{0} M_{i}$ and so $\operatorname{dim}\left(M_{i}\right) \leq \operatorname{dim}\left(X_{0}\right) \operatorname{dim}\left(Y_{0} M_{i}\right) \leq$ $e \operatorname{dim}\left(Y_{0} M_{i}\right)$, this proves the first inequality. The second follows from the following inclusions: $Y_{0} M_{i}=Y_{0} A_{i} M_{0} \subseteq B_{c i} Y_{0} M_{0}=M_{c i}^{\prime}$.

By (5), GK $\left({ }_{A} M\right) \leq \mathrm{GK}\left({ }_{B} M^{\prime}\right)$. By symmetry, we also have the inequality $\mathrm{GK}\left({ }_{B} M^{\prime}\right) \leq$ $\operatorname{GK}\left({ }_{A} M^{\prime \prime}\right)$ where $M^{\prime \prime}:=X \otimes_{B} Y \otimes_{A} M=A X_{0} Y_{0} M_{0}$, and $\left\{M_{i}^{\prime \prime}:=A_{i} X_{0} Y_{0} M_{0}\right\}$ is the standard filtration on $M^{\prime \prime}$. Now, $1_{A} \in X_{0} Y_{0}$ implies $M_{i}=A_{i} M_{0}=A_{i} 1_{A} M_{0} \subseteq A_{i} X_{0} Y_{0} M_{0}=$ $M_{i}^{\prime \prime}$. On the other hand, $M_{i}^{\prime \prime} \subseteq X_{0} B_{c i} Y_{0} M_{0} \subseteq X_{0} Y_{0} A_{c^{2} i} M_{0}=X_{0} Y_{0} M_{c^{2} i}$. Summarizing, we have

$$
\operatorname{dim}\left(M_{i}\right) \leq \operatorname{dim}\left(M_{i}^{\prime \prime}\right) \leq e^{2} \operatorname{dim}\left(M_{c^{2} i}\right), \quad i \geq 0 .
$$

Hence, $\operatorname{GK}\left({ }_{A} M\right)=\mathrm{GK}\left({ }_{A} M^{\prime \prime}\right)$ which implies $\mathrm{GK}\left({ }_{A} M\right)=\mathrm{GK}\left({ }_{B} M^{\prime}\right)$ (since $\mathrm{GK}\left({ }_{A} M\right) \leq$ $\left.\mathrm{GK}\left({ }_{B} M^{\prime}\right) \leq \mathrm{GK}\left({ }_{A} M^{\prime \prime}\right)=\mathrm{GK}\left({ }_{B} M\right)\right)$, as required.

Corollary 2.1 Let $A$ be a simple finitely generated algebra and an algebra $B$ be Morita equivalent to $A$. Then

$$
\operatorname{GK}(M) \geq h_{A} \geq \frac{\operatorname{GK}(A)}{\operatorname{fdim}(A)+\max \{\operatorname{fdim}(A), 1\}}
$$

for all nonzero finitely generated B-modules $M$. 
Proof. The algebras $A$ and $B$ are Morita equivalent, hence $\mathrm{GK}(A)=\mathrm{GK}(B), B$ is a simple finitely generated algebra, and $\operatorname{fdim}(A)=\operatorname{fdim}(B)$ (Theorem 1.3). By Theorems 1.2 and 1.6 .

$$
\operatorname{GK}(M) \geq h_{B}=h_{A} \geq \frac{\operatorname{GK}(B)}{\operatorname{fdim}(B)+\max \{\operatorname{fdim}(B), 1\}}=\frac{\operatorname{GK}(A)}{\operatorname{fdim}(A)+\max \{\operatorname{fdim}(A), 1\}}
$$

for any nonzero finitely generated $B$-module $M$.

Corollary 2.2 Let $A$ be a simple finitely generated algebra, $e=e^{2}$ be a nonzero idempotent of $A$, and $B:=e A e$. Then

$$
\operatorname{GK}(M) \geq h_{A} \geq \frac{\operatorname{GK}(A)}{\operatorname{fdim}(A)+\max \{\operatorname{fdim}(A), 1\}}
$$

for any nonzero finitely generated $B$-module $M$.

Proof. The algebra $B$ is Morita equivalent to $A,\left[8\right.$ (the Morita context $\left(\begin{array}{cc}A & A e \\ e A & e A e\end{array}\right)$ provides a Morita equivalence since $e A A e=e A e$ and $A e e A=A$, since $A$ is simple and $e \neq 0)$. Now, applying Corollary 2.1] we have the result.

Corollary 2.3 Let $A$ be a simple finitely generated algebra, $G$ be a finite group of algebra automorphisms of $A$ such that $|G|^{-1} \in A, A^{G}$ be the fixed algebra. Then

$$
\mathrm{GK}(M) \geq h_{A} \geq \frac{\operatorname{GK}(A)}{\operatorname{fdim}(A)+\max \{\operatorname{fdim}(A), 1\}}
$$

for any nonzero finitely generated $A^{G}$-module $M$.

Proof. The algebras $A$ and $A^{G}$ are Morita equivalent [8], 7.8.6. Now, use Corollary 2.1.

\section{Acknowledgements}

The authors would like to thank Y. Berest, K. Brown, O. Chalykh, I. Gordon and A. Veselov for interesting discussions.

\section{References}

[1] V. V. Bavula, Filter dimension of algebras and modules, a simplicity criterion for generalized Weyl algebras. Comm. Algebra 24 (1996), no. 6, 1971-1992.

[2] V. V. Bavula, Krull, Gelfand-Kirillov, and filter dimensions of simple affine algebras. J. Algebra 206 (1998), no. 1, 33-39. 
[3] V. V. Bavula, Filter dimension, Handbook of Algebra, vol. 4 (Ed. by M. Hazewinkel), (2006), 77-105.

[4] Y. Berest, P. Etingof, and V. Ginzburg, Cherednik algebras and differential operators on quasi-invariants. Duke Math. J. 118 (2003), no. 2, 279-337.

[5] O. A. Chalykh and A. P. Veselov, Commutative rings of partial differential operators and Lie algebras. Comm. Math. Phys. 126 (1990), no. 3, 597-611.

[6] M. Feigin and A. P. Veselov, Quasi-invariants of Coxeter groups and $m$-harmonic polynomials. Int. Math. Res. Not. (2002), no. 10, 521-545.

[7] G. Krause and T. Lenagan, Growth of algebras and Gelfand-Kirillov dimension. Revised edition. Graduate Studies in Mathematics, 22. American Mathematical Society, Providence, RI, 2000.

[8] J. C. McConnell and J. C. Robson, Noncommutative Noetherian rings, Wiley, Chichester, 1987.

Department of Pure Mathematics

University of Sheffield

Hicks Building

Sheffield S3 7RH

UK

email: v.bavula@sheffield.ac.uk 